\title{
Erratum to: Design and Rationale of Japanese Evaluation Between Formula of Azelnidipine and Amlodipine Add on Olmesartan to Get Antialbuminuric Effect Study (J-FLAG)
}

\author{
Evaluation of the Antialbuminuric Effects between Calcium Channel Blocker \\ with Sympatholytic Action in Hypertensive Patients with Diabetes and Albuminuria
}

\author{
Katsuyuki Ando • Masakazu Haneda • Sadayoshi Ito • \\ Naoki Kashihara • Koichi Node • Masaomi Nangaku • \\ Tatsuo Shimosawa • Junji Kishimoto • Toshiro Fujita
}

Published online: 7 July 2011

(C) Springer Science+Business Media, LLC 2011

Erratum to: Cardiovasc Drugs Ther

DOI 10.1007/s10557-011-6309-5

The online version of the original article can be found at http://dx.doi. org/10.1007/s10557-011-6309-5.

K. Ando $\cdot$ M. Nangaku $\cdot$ T. Fujita $(\triangle)$

Department of Nephrology and Endocrinology,

Faculty of Medicine, University of Tokyo,

7-3-1 Hongo, Bunkyo-ku,

Tokyo 113-8655, Japan

e-mail: fujita-dis@h.u-tokyo.ac.jp

T. Shimosawa

Department of Clinical Laboratory, Faculty of Medicine, University of Tokyo,

7-3-1 Bunkyo-ku, Hongo,

Tokyo 113-8655, Japan

\section{Haneda}

Division of Metabolism and Biosystemic Science,

Department of Medicine, Asahikawa Medical University,

1-1, 2-1 Midorigaokahigashi,

Asahikawa, Hokkaido 078-8510, Japan
S. Ito

Division of Nephrology, Endocrinology, and Vascular Medicine,

Department of Internal Medicine,

Tohoku University Graduate School of Medicine,

1-1 Seiryo-machi, Aoba-ku,

Sendai 980-8574, Japan

N. Kashihara

Division of Nephrology,

Department of Internal Medicine,

Kawasaki Medical School,

557 Matsushima,

Kurashiki, Okayama 701-0192, Japan

K. Node

Department of Cardiovascular Medicine,

Saga University,

Nabeshima 5-1-1,

Saga 849-8501, Japan

J. Kishimoto

Center for Clinical and Translational Research,

Kyushu University Hospital,

3-1-1 Maidashi, Higashi-ku,

Fukuoka 812-8582, Japan 
Figure:

The doses of azelnidipine in figure are not $2.5-5 \mathrm{mg} /$ day but $8-16 \mathrm{mg} /$ day as shown in the below.

Fig. 1 Design of the J-FLAG (Japanese evaluation between FormuLa of Azelnidipine and amlodipine add on olmesartan to Get antialbuminuric effect study) trial

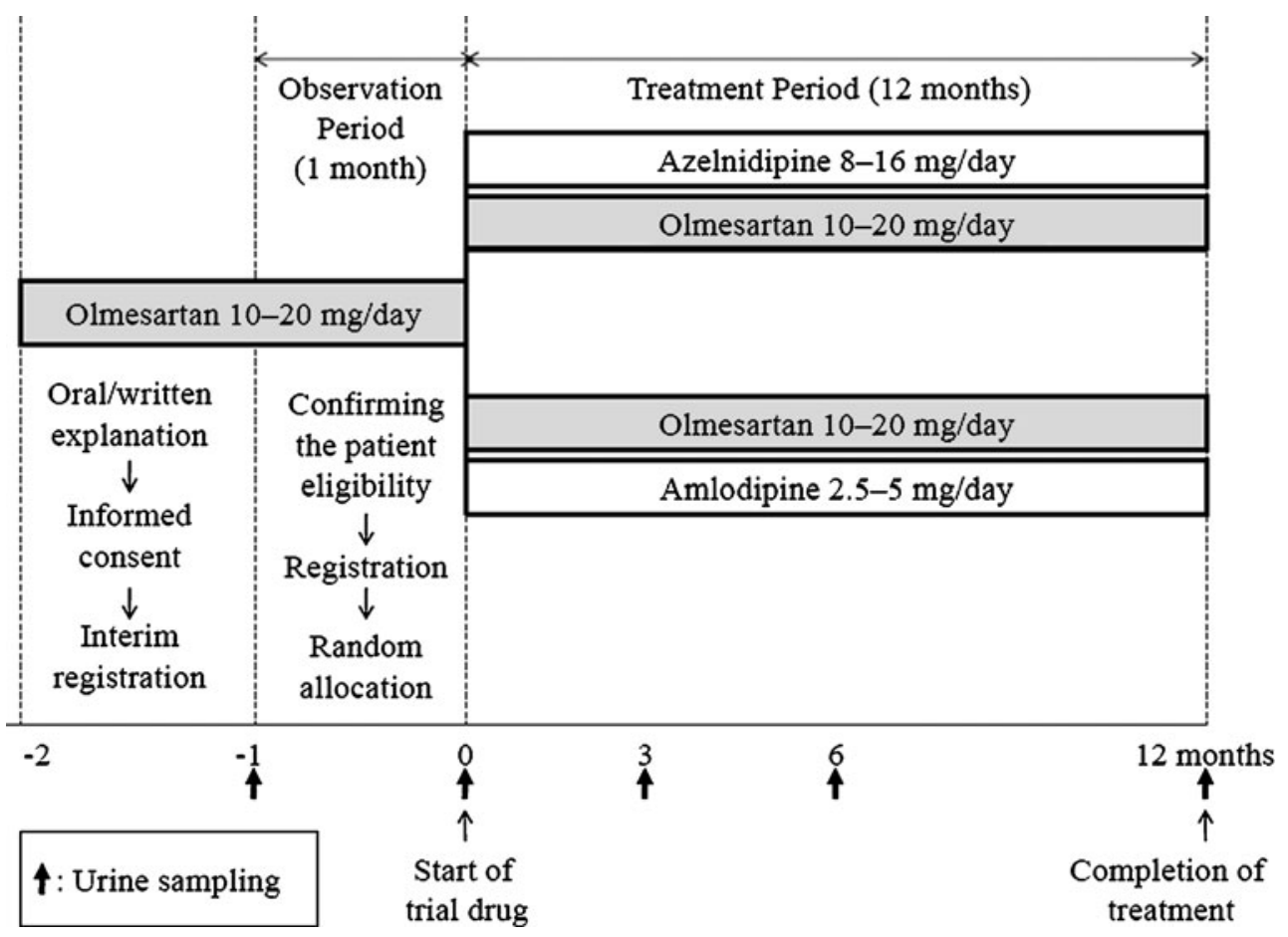

